\title{
REPRÉSENTATION ASYMPTOTIQUE DES FONCTIONS DE MATHIEU ET DES FONCTIONS SPHÉROIDALES, II
}

\author{
BY \\ ROBERT SIPS \\ INTRODUCTION
}

Dans un travail antérieur [1], nous avons étudié la représentation asymptotique des fonctions de Mathieu et des fonctions sphéroidales lorsque le paramètre prend des valeurs élevées.

Les représentations obtenues montrent que les fonctions en question n'acquièrent dans ce cas des valeurs appréciables que dans le voisinage immédiat du maximum absolu de la fonction. Dans cette région, les formules asymptotiques donnent des valeurs remarquablement exactes, l'exactitude étant d'autant meilleure que le nombre de zéros de la fonction est plus petit.

Cependant, au fur et à mesure que l'on s'éloigne du maximum absolu, les valeurs fournies par les formules asymptotiques deviennent de plus en plus inexactes et finissent par ne plus même avoir l'ordre de grandeur correct. De plus, les valeurs dans cette région sont extrêmement faibles (de l'ordre de $e^{-\lambda c}$, alors que d'après les formules asymptotiques, cet ordre serait $e^{-1 / 2 \lambda c}$ ) et il devient matériellement impossible de les calculer au moyen des développements en série habituellement en usage.

Dans les présent travail, nous donnons des formules asymptotiques qui permettent le calcul direct des valeurs des fonctions dans le domaine où les formules établies antérieurement sont inutilisables.

\section{Fonctions de Mathieu}

1. Les fonctions de Mathieu sont les solutions périodiques, de période $2 \pi$, de l'équation différentielle

$$
\frac{d^{2} y}{d \eta^{2}}+\left(a-\frac{(\lambda c)^{2}}{2} \cos 2 \eta\right) y=0
$$

Dans ce qui suit, nous représenterons, de la manière habituelle, par $c e_{n}(\eta)$ et $s e_{n}(\eta)$ celles de ces solutions qui, pour $\lambda c=0$, se réduisent respectivement à $\cos n \eta$ et $\sin n \eta$, et qui, de plus, vérifient la condition de normalisation

$$
\int_{0}^{2 \pi} c e_{n}^{2}(\eta) d \eta=\int_{0}^{2 \pi} s e_{n}^{2}(\eta) d \eta=\pi
$$

La valeur correspondante de la constante de séparation sera représentée par $a_{n}$ ou $b_{n}$.

Received by the editors April 29, 1957. 
On sait que, lorsque $\lambda c$ est très grand et que $\eta$ est compris 0 et $\pi, c e_{n}(\eta)$ et $s e_{n}(\eta)$ sont représentés asymptotiquement par les formules suivantes

$$
\begin{aligned}
c e_{n}(\eta) \sim & C\left[D_{n}(\alpha)-\frac{1}{2 \lambda c}\left(\frac{D_{n+4}(\alpha)}{16}+\frac{D_{n+2}(\alpha)}{4}-\frac{n(n+1)}{4} D_{n-2}(\alpha)\right.\right. \\
& \left.+\frac{n(n-1)(n-2)(n-3)}{16} D_{n-4}(\alpha)\right)+\frac{1}{(2 \lambda c)^{2}}\left(\frac{D_{n+8}(\alpha)}{512}+\frac{D_{n+6}(\alpha)}{64}\right. \\
& -\frac{n+2}{16} D_{n+4}(\alpha)+\frac{n^{2}-25 n-36}{64} D_{n+2}(\alpha) \\
& +\frac{n(n-1)\left(-n^{2}-27 n+10\right)}{64} D_{n-2}(\alpha) \\
& +\frac{n(n-1)^{2}(n-2)(n-3)}{16} D_{n-4}(\alpha) \\
& -\frac{n(n-1) \cdots(n-5)}{64} D_{n-6}(\alpha) \\
& \left.\left.+\frac{n(n-1) \cdots(n-7)}{512} D_{n-8}(\alpha)\right)+O\left(\lambda c^{-3}\right)\right]
\end{aligned}
$$

où $\alpha=(2 \lambda c)^{1 / 2} \cos \eta$ et où la constante $C$ a la valeur

$$
C=\frac{(\pi \lambda c)^{1 / 4}}{2^{1 / 2}(n !)^{1 / 2}}\left[1+\frac{c_{1}}{2 \lambda c}+\frac{c_{2}}{(2 \lambda c)^{2}}+\cdots\right]
$$

avec

$$
\begin{aligned}
c_{1}= & n+1 / 2, \\
c_{2}= & \frac{(n+1) \cdots(n+4)+n(n-1) \cdots(n-3)}{256} \\
& -3 \frac{(n+1)(n+2)+n(n-1)}{16} \\
& +9 \frac{2 n^{2}+2 n+1}{8} .
\end{aligned}
$$

Nous avons donné, dans [1], ces formules sous une forme un peu différente.

La représentation asymptotique (3) donne la valeur des fonctions $c e_{n}$ et $s e_{n}$, lorsque $c$ est assez grand et $n$ assez petit, avec une exactitude remarquable pour les valeurs de $\eta$ contenues dans un domaine assez étroit voisin de $\eta=\pi / 2$, domaine où, en fait, les fonctions prennent seulement des valeurs 
numériques appréciables. Pour les autres valeurs de $\eta$, et en particulier pour $\eta=0$ (et $=\pi$ ) la formule (3) donne des valeurs totalement inexactes qui n'ont même pas l'ordre de grandeur correct.

Nous donnerons ci-dessous des formules utilisables dans cette dernière région.

2. Les fonctions $c e_{n}$ et $s e_{n}$ satisfont à deux groupes d'équations intégrales linéaires

a.

$$
\begin{aligned}
& c e_{n}(\eta)=\lambda_{n} \int_{-\pi}^{\pi} e^{i \lambda c \cos \eta \cos \eta_{j} c e_{n}\left(\eta_{0}\right) d \eta_{0},} \\
& s e_{n}(\eta)=\mu_{n} \int_{-\pi}^{\pi} e^{i \lambda c \cos \eta \cos \eta_{0}} \sin \eta \sin \eta_{0} s e_{n}\left(\eta_{0}\right) d \eta_{0} .
\end{aligned}
$$

b.

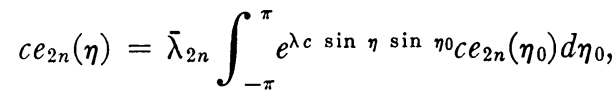

$$
\begin{aligned}
& s e_{2 n+1}(\eta)=\bar{\mu}_{2 n+1} \int_{-\pi}^{\pi} e^{\lambda c \sin \eta \sin \eta_{0}} s e_{2 n+1}\left(\eta_{0}\right) d \eta_{0}, \\
& s e_{2 n}(\eta)=\bar{\mu}_{2 n} \int_{-\pi}^{\pi} e^{\lambda c \sin \eta \sin \eta_{0}} \cos \eta \cos \eta_{0} s e_{2 n}\left(\eta_{0}\right) d \eta_{0}, \\
& c e_{2 n+1}(\eta)=\bar{\lambda}_{2 n+1} \int_{-\pi}^{\pi} e^{\lambda c \sin \eta \sin \eta_{0}} \cos \eta \cos \eta_{0} c e_{2 n+1}\left(\eta_{0}\right) d \eta_{0} .
\end{aligned}
$$

De ces équations on déduit immédiatement que

$$
\begin{aligned}
c e_{2 n}(0) & =\frac{\bar{\lambda}_{2 n}}{\lambda_{2 n}} c e_{2 n}\left(\frac{\pi}{2}\right), \\
c e_{2 n+1}(0) & =-\frac{i}{\lambda c} \cdot \frac{\bar{\lambda}_{2 n+1}}{\lambda_{2 n+1}} c e_{2 n+1}^{\prime}\left(\frac{\pi}{2}\right), \\
s e_{2 n}^{\prime}(0) & =-\frac{i \bar{\mu}_{2 n}}{\mu_{2 n}} s e_{2 n}^{\prime}\left(\frac{\pi}{2}\right), \\
s e_{2 n+1}^{\prime}(0) & =\lambda c \frac{\bar{\mu}_{2 n+1}}{\mu_{2 n+1}} s e_{2 n+1}\left(\frac{\pi}{2}\right),
\end{aligned}
$$

de sorte que le problème de la détermination de la valeur asymptotique des fonctions $c e_{n}$ et $s e_{n}$ pour $\eta=0$ se ramène à la détermination de la représentation asymptotique des constantes caractéristiques $\lambda_{n}, \mu_{n}, \bar{\lambda}_{n}, \bar{\mu}_{n}$.

Nous avons déja donné précédemment [2] les valeurs asymptotiques des constantes $\lambda_{n}$ et $\mu_{n}$, que nous reproduisons ci-dessous 


$$
\begin{aligned}
\lambda_{n} & =i^{n} \frac{(2 \lambda c)^{1 / 2}}{4(\pi)^{1 / 2}}\left[1+\frac{2 n+1}{4 \lambda c}+\frac{16 n^{2}+16 n+7}{32(\lambda c)^{2}}+\cdots\right]^{-1}, \\
\mu_{n+1} & =i^{n} \frac{(2 \lambda c)^{1 / 2}}{4(\pi)^{1 / 2}}\left[1-\frac{2 n+1}{4 \lambda c}-\frac{8 n^{2}+8 n+5}{32(\lambda c)^{2}}-\cdots\right]^{-1}
\end{aligned}
$$

et nous allons maintenant rechercher les représentations correspondantes des constantes $\bar{\lambda}_{n}$ et $\bar{\mu}_{n}$.

3. Considérons pour commencer l'équation ( 7 ) et supposons $0<\eta \leqq \pi$, de sorte que $\sin \eta>0$. Alors $\lambda c \sin \eta \sin \eta_{0}$ sera positif pour $0<\eta_{0} \leqq \eta$ et négatif pour $-\pi \leqq \eta_{0}<0$. D'autre part, pour $\lambda c$ très grand, $c e_{2 n}\left(\eta_{0}\right)$ ne prend des valeurs appréciables qu'aux environs de $\eta_{0}=\pi / 2$. Il en résulte que le partie de l'intégrale prise entre $-\pi$ et 0 sera de l'ordre de $e^{-2 \lambda c}$ par rapport à la partie prise entre 0 et $\pi$ et pourra en conséquence être négligée.

On aura donc asymptotiquement

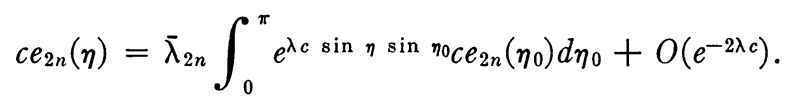

De même, si $\sin \eta<0$, on aura

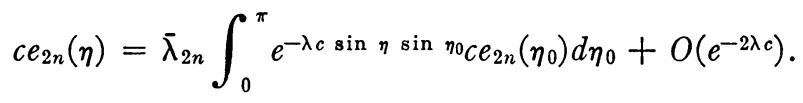

On peut montrer de la même manière que, pour $0<\eta \leqq \pi$

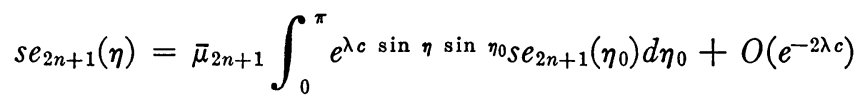

et, de plus, comme les représentations asymptotiques de $c e_{2 n}$ et $s e_{2 n+1}$ sont identiques pour $0 \leqq \eta \leqq \pi / 2$, on aura également l'égalité asymptotique suivante

$$
\bar{\lambda}_{2 n}=\bar{\mu}_{2 n+1} .
$$

4. Posons, dans l'équation (17)

$$
\alpha=(2 \lambda c)^{1 / 2} \cos \eta, \quad \alpha_{0}=(2 \lambda c)^{1 / 2} \cos \eta_{0}
$$

ce qui la transforme en

$$
y_{2 n}(\alpha)=\frac{\bar{\lambda}_{2 n}}{(2 \lambda c)^{1 / 2}} \int_{-(2 \lambda c)^{1 / 2}}^{(2 \lambda c)^{1 / 2}} \exp \left(\lambda c\left(1-\alpha^{2} / 2 \lambda c\right)^{1 / 2}\left(1-\alpha_{0}^{2} / 2 \lambda c\right)^{1 / 2}\right)
$$

$$
\frac{y_{2 n}\left(\alpha_{0}\right)}{\left(1-\frac{\alpha_{0}^{2}}{2 \lambda c}\right)^{1 / 2}} d \alpha_{0}
$$


où nous avons posé

$$
c e_{2 n}(\eta)=C y_{2 n}(\alpha)
$$

c'est-à-dire que $y_{n}(\alpha)$ représente la quantité entre crochets dans (3). $\lambda c$ étant très grand, nous pourrons prendre comme limites d'intégration $-\infty$ et $\infty$. En faisant encore $\alpha=0$, nous obtenons finalement

$$
y_{2 n}(0)=\frac{\bar{\lambda}_{2 n}}{(2 \lambda c)^{1 / 2}} e^{-\lambda c} \int_{-\infty}^{\infty} e^{-\alpha^{2} / 4} \Sigma(\alpha) y_{2 n}(\alpha) d \alpha
$$

en posant encore, pour abréger

$$
\Sigma(\alpha)=\frac{\exp \left(\lambda c\left(\left(1-\frac{\alpha^{2}}{2 \lambda c}\right)^{1 / 2}-1+\frac{\alpha^{2}}{4}\right)\right)}{\left(1-\frac{\alpha^{2}}{2 \lambda c}\right)^{1 / 2}} .
$$

En développant $\Sigma(\alpha)$ suivant les puissances décroissantes de $\lambda c$ on trouve

$$
\begin{aligned}
\Sigma(\alpha)=1 & +\frac{1}{2^{5} \lambda c}\left(2^{3} \alpha^{2}-\alpha^{4}\right)+\frac{1}{2^{11}(\lambda c)^{2}}\left(2^{6} \cdot 3 \cdot \alpha^{4}-2^{5} \alpha^{6}+\alpha^{8}\right) \\
& +\frac{1}{2^{16} \cdot 3 \cdot(\lambda c)^{3}}\left(2^{9} \cdot 3 \cdot 5 \alpha^{6}-2^{5} \cdot 3^{2} \cdot 5 \alpha^{8}+2^{3} \cdot 3^{2} \cdot \alpha^{10}-\alpha^{12}\right) \\
& +\frac{1}{2^{23} \cdot 3 \cdot(\lambda c)^{4}}\left(2^{12} \cdot 3 \cdot 5 \cdot 7 \alpha^{8}-2^{12} \cdot 3 \cdot 7 \cdot \alpha^{10}\right. \\
& \left.+2^{7} \cdot 3 \cdot 7 \alpha^{12}-2^{6} \cdot 3 \alpha^{14}+\alpha^{16}\right)+\cdots
\end{aligned}
$$

En introduisant dans (23) cette valeur de $\Sigma(\alpha)$ ainsi que la représentation asymptotique (1) (dans laquelle il nous a fallu encore tenir compte en plus des termes en $D_{0}(\alpha) /(2 \lambda c)^{3}$ s'ils existent) on obtient finalement, après un calcul simple, mais assez fastidieux, les valeurs suivantes

$$
\begin{aligned}
& \bar{\lambda}_{0}=\bar{\mu}_{1}=\frac{(2 \lambda c)^{1 / 2}}{(2 \pi)^{1 / 2}} e^{-\lambda c}\left(1-\frac{1}{8 \lambda c}-\frac{3}{64(\lambda c)^{2}}-\cdots\right), \\
& \bar{\lambda}_{2}=\bar{\mu}_{3}=4 \frac{(2 \lambda c)^{3 / 2}}{(2 \pi)^{1 / 2}} e^{-\lambda c}\left(1-\frac{11}{8 \lambda c}-\frac{19}{32(\lambda c)^{2}}-\cdots\right) .
\end{aligned}
$$

Le terme dominant du développement asymptotique de $\bar{\lambda}_{2 n}$ a la forme suivante

$$
\bar{\lambda}_{2 n} \sim A_{2 n} e^{-\lambda c} \frac{(2 \lambda c)^{n+1 / 2}}{(2 \pi)^{1 / 2}}
$$


Nous avons calculé la valeur de la constante $A_{2 n}$ pour $n=0,1,2,3$ et avons obtenu les valeurs suivantes

$$
\begin{array}{ll}
A_{0}=1, & A_{2}=4, \\
A_{4}=16 / 3, & A_{6}=64 / 15 .
\end{array}
$$

On constate que pour ces valeurs de $n$

$$
A_{2 n}=\frac{2^{3 n} n !}{2 n !} \text {. }
$$

Il parait probable, bien que nous n'ayons pas jusqu'ici réussi à le démontrer, que cette formule est générale. Si cette hypothèse est exacte, on aura

$$
\bar{\lambda}_{2 n} \sim \frac{2^{3 n} n !}{2 n !} e^{-\lambda c} \frac{(2 \lambda c)^{n+1 / 2}}{(2 \pi)^{1 / 2}} .
$$

5. Dans le cas des fonctions $c e_{2 n+1}$ et $s e_{2 n}$, nous utiliserons, de la même manière que plus haut, les équations intégrales (9) et (10). En admettant que $0<\eta \leqq \pi / 2$, il suffira encore une fois de considérer uniquement l'intégrale prise entre 0 et $\pi$, et on constatera de plus que, asymptotiquement

$$
\bar{\lambda}_{2 n+1}=\bar{\mu}_{2 n+2} \text {. }
$$

La valeur de $\bar{\lambda}_{2 n+1}$ sera donnée par la relation suivante, analogue à (23)

$$
y_{2 n+1}^{\prime}(0)=(\lambda c)^{-3 / 2} \bar{\lambda}_{2 n+1} e^{\lambda c} \int_{-\infty}^{\infty} e^{-\alpha^{2} / 4} \alpha \Sigma(\alpha) y_{2 n+1}(\alpha) d \alpha .
$$

En introduisant dans cette relation le développement (25) de $\Sigma(\alpha)$ et la représentation asymptotique (1) (dans laquelle il faudra cette fois ci tenir compte des termes en $D_{1}(\alpha) /(2 \lambda c)^{3}$, s'ils existent), on trouve, après un calcul simple, mais assez laborieux, les valeurs suivantes

$$
\begin{aligned}
\bar{\lambda}_{1} & =\bar{\mu}_{2} \\
& =\frac{(2 \lambda c)^{3 / 2}}{(2 \pi)^{1 / 2}} e^{-\lambda c}\left(1-\frac{3}{8 \lambda c}-\frac{15}{64(\lambda c)^{2}}-\cdots\right), \\
\bar{\lambda}_{3} & =\bar{\mu}_{4} \\
& =4 / 3 \frac{(2 \lambda c)^{5 / 2}}{(2 \pi)^{1 / 2}} e^{-\lambda c}\left(1-\frac{17}{8 \lambda c}-\frac{87}{128(\lambda c)^{2}}-\cdots\right) .
\end{aligned}
$$

Le terme dominant du développement asymptotique de $\bar{\lambda}_{2 n+1}$ a la forme suivante 


$$
\bar{\lambda}_{2 n+1}=B_{2 n+1} e^{-\lambda c} \frac{(2 \lambda c)^{n+3 / 2}}{(2 \pi)^{1 / 2}}
$$

Nous avons calculé la valeur de la constante $B_{2 n+1}$ pour $n=0,1$ et 2 , et obtenons

$$
\begin{aligned}
& B_{1}=1, \\
& B_{3}=4 / 3, \\
& B_{5}=16 / 15 .
\end{aligned}
$$

On constate que pour ces valeurs de $n$

$$
B_{2 n+1}=\frac{2^{3 n} n !}{2 n+1 !}
$$

Il parait fort probable que cette formule, comme (29), est générale. On aura donc dans ce cas

$$
\bar{\lambda}_{2 n+1} \sim \frac{2^{3 n} n !}{2 n+1 !} e^{-\lambda c} \frac{(2 \lambda c)^{n+3 / 2}}{(2 \pi)^{1 / 2}} .
$$

6. Comme vérification numérique des formules obtenues ci-dessus, nous avons comparé les valeurs exactes, pour $\lambda c=12$, de $c e_{0}(0), c e_{1}(0), c e_{2}(0)$, $c e_{3}(0)$ et de $s e_{1}^{\prime}(0), s e_{2}^{\prime}(0), s e_{3}^{\prime}(0)$, obtenues à partir des tables de E. L. Ince [3], avec les valeurs asymptotiques de ces mêmes quantités calculées au moyen des formules (11), (12), (13) et (14). Les valeurs des fonctions pour $\eta=\pi / 2$ ont été calculées au moyen de (3) et (4) et celles de $\lambda_{n}, \mu_{n}$ et $\bar{\lambda}_{n}, \bar{\mu}_{n}$ respectivement en utilisant (15), (16) et (26), (27), (33) et (34). Les résultats sont donnés dans le tableau ci-dessous:

\begin{tabular}{l|c|c}
\hline & Valeur exacte. & Valeur approchée. \\
\hline$c e_{0}(\pi / 2)$ & 1,737833 & 1,73769 \\
$c e_{0}(0)$ & 0,0000306 & 0,0000300 \\
$s e_{1}(\pi / 2)$ & 1,737833 & 1,73769 \\
$s e_{1}^{\prime}(0)$ & 0,0003508 & 0,000350 \\
$c e_{1}^{\prime}(\pi / 2)$ & $-8,226091$ & $-8,22597$ \\
$c e_{1}(0)$ & 0,0002968 & 0,0002936 \\
$s e_{2}^{\prime}(\pi / 2)$ & $-8,226091$ & $-8,22597$ \\
$s e_{2}^{\prime}(0)$ & 0,0030894 & 0,0030705 \\
$c e_{2}(\pi / 2)$ & $-1,182423$ & $-1,18565$ \\
$c e_{2}(0)$ & 0,0019718 & 0,0019465 \\
$s e_{3}(\pi / 2)$ & $-1,182427$ & $-1,18565$ \\
$s e_{3}^{\prime}(0)$ & 0,0182617 & 0,018336 \\
$c e_{3}^{\prime}(\pi / 2)$ & 9,388572 & 9,17212 \\
$c e_{3}(0)$ & 0,0103295 & 0,010725 \\
\hline
\end{tabular}


On remarquera que, comme toujours, l'exactitude diminue notablement pour une même valeur de $\lambda c$, lorsque $n$ augmente.

Comme autre exemple numérique, nous avons calculé la valeur de $c e_{0}(0)$ pour $\lambda c=80$. Cette valeur a déja été calculée précédemment par $G$. Blanch [4] par une méthode sur laquelle nous reviendrons plus loin. G. Blanch trouve que

$$
c e_{0}(0)=1,44 \cdot 10^{-34}
$$

tandis que nos formules asymptotiques donnent

$$
c e_{0}(0)=1,43782 \cdot 10^{-34} \text {. }
$$

On voit que l'accord est excellent et, en fait, la seconde valeur est certainement plus exacte que la première.

7. Ayant ainsi obtenu la représentation asymptotique de $c e_{n}(0)$ et de $s e_{n}^{\prime}(0)$, il nous faut maintenant rechercher la valeur des fonctions $c e_{n}(\eta)$ et $s e_{n}(\eta)$ elles-mêmes aux environs de $\eta=0$.

Nous utiliserons dans ce but les équations intégrales (7), (8), (9) et (10). Comme nous l'avons déja dit plus haut, lorsque $\lambda c$ est grand, les fonctions $c e_{n}$ et $s e_{n}$ ne prennent des valeurs appréciables qu'aux environs de $\eta=\pi / 2$, c'est-à-dire précisément dans le domaine d'utilisation de la représentation asymptotique (3).

Si donc nous introduisons ce développement dans les équations intégrales, le résultat sera certainement une bonne approximation des fonctions $c e_{n}$ et $s e_{n}$ pour toutes les valeurs de $\eta$.

Considérons, pour commencer, les fonctions $c e_{2 n}$ et $s e_{2 n+1}$ et, pour simplifier, nous n'utiliserons que le premier terme de (3). Donc, entre 0 et $\pi$,

$$
c e_{2 n}(\eta) \sim s e_{2 n+1}(\eta) \sim C D_{2 n}(\alpha)
$$

et entre $\pi$ et $2 \pi$,

$$
c e_{2 n}(\eta) \sim-s e_{2 n+1}(\eta) \sim C D_{2 n}(\alpha) .
$$

On peut par conséquent écrire

$$
\left.\begin{array}{l}
c e_{2 n}(\eta) \\
s e_{2 n+1}(\eta)
\end{array}=\begin{array}{l}
\bar{\lambda}_{2 n} \\
\bar{\mu}_{2 n+1}
\end{array}\right\} C \int_{-\pi / 2}^{\pi / 2}\left(e^{\lambda c \sin \eta \sin \eta_{0}} \pm e^{-\lambda c \sin \eta \sin \eta_{0}}\right) D_{2 n}\left((2 \lambda c)^{1 / 2} \cos \eta_{0}\right) d \eta_{0} .
$$

La fonction à intégrer passant, pour $\eta_{0}=0$, par un maximum très accentué lorsque $\lambda c$ est très grand, nous obtendrons une valeur approchée de l'intégrale définie en remplaçant partout $\sin \eta_{0}$ et $\cos \eta_{0}$ respectivement pas $\eta_{0}$ et $1-\eta_{0}^{2} / 2$ et en prenant comme limites d'intégration $\pm \infty$. Il vient ainsi

$$
\int_{-\infty}^{\infty}\left(e^{\lambda c \sin \eta\left(1-\eta_{0} / 2\right)} \pm e^{-\lambda c \sin \eta\left(1-\eta_{0}{ }^{2} / 2\right)}\right) e^{-\lambda c \eta_{0}{ }^{2} / 2} H e_{2 n}\left((2 \lambda c)^{1 / 2} \eta_{0}\right) d \eta_{0}
$$


où $H e_{2 n}$ désigne le polynôme d'Hermite. Ceci peut encore s'écrire

$$
e^{\lambda c \sin \eta} \int_{-\infty}^{\infty} e^{-2 \lambda c \cos ^{2}(\pi / 4-\eta / 2) \cdot \eta_{0}{ }^{2} / 2} H e_{2 n}\left((2 \lambda c)^{1 / 2} \eta_{0}\right) d \eta_{0}
$$

$$
\pm e^{-\lambda c \sin \eta} \int_{-\infty}^{\infty} e^{-2 \lambda c \sin ^{2}(\pi / 4-\eta / 2) \cdot \eta_{0}{ }^{2} / 2} H e_{2 n}\left((2 \lambda c)^{1 / 2} \eta_{0}\right) d \eta_{0}
$$

On sait d'autre part que

$$
\int_{-\infty}^{\infty} e^{-x^{2} / 2} H e_{2 n}(\alpha x) d x=(2 \pi)^{1 / 2} \frac{2 n !}{2^{n} n !}\left(\alpha^{2}-1\right)^{n}
$$

et par conséquent, finalement

$$
\begin{aligned}
& \left.\begin{array}{l}
c e_{2 n}(\eta)=\bar{\lambda}_{2 n} \\
s e_{2 n+1}(\eta)=\bar{\mu}_{2 n+1}
\end{array}\right\} C \frac{(2 \pi)^{1 / 2}}{(2 \lambda c)^{1 / 2}} \cdot \frac{2^{n+1} 2 n !}{n ! \cos ^{2 n+1} \eta} \\
& \cdot\left[e^{\lambda c \sin \eta} \cos ^{4 n+1}(\pi / 4+\eta / 2) \pm e^{-\lambda c \sin \eta} \sin ^{4 n+1}(\pi / 4+\eta / 2)\right] \text {. }
\end{aligned}
$$

Nous retrouvons ainsi le premier terme d'un développement asymptotique des fonctions de Mathieu du à S. Goldstein [5], mais avec en plus un coefficient numérique permettant directement le raccordement avec le développement (3).

S. Goldstein avait obtenu le développement (42) directement à partir de l'équation différentielle (1). Son procédé, qui est sans aucun doute le plus commode pour obtenir les termes suivants du développement, ne peut cependant pas fournir la valeur du coefficient numérique correspondant à la normalisation (2) puisque (42) devient infini pour $\eta=\pi / 2$.

Il est facile de voir pourquoi le développement (42) n'est pas utilisable pour $\eta=\pi / 2$. En effet, pour cette valeur, la seconde intégrale définie de (40) devient infinie. En fait, un calcul plus exact montre que la seconde intégrale définie de (38) est $O\left(e^{-2 \lambda c}\right)$ pour $\eta=\pi / 2$ et est donc négligeable. Il reste donc uniquement la première. En la calculant, on trouve qu'avec l'approximation utilisée plus haut, elle s'annule identiquement, sauf pour $n=0$.

Ceci montre que l'approximation fournie par l'utilisation de la méthode du col est insuffisante pour $\eta$ voisin de $\pi / 2$.

On arrive évidemment à la même conclusion si l'on considère les fonctions $c e_{2 n+1}(\eta)$ et $s e_{2 n}(\eta)$. On trouve, pour $\eta$ voisin de 0 :

$$
\begin{aligned}
& \left.\begin{array}{l}
c e_{2 n+1}(\eta)=\bar{\lambda}_{2 n+1} \\
s e_{2 n+2}(\eta)=\bar{\mu}_{2 n+2}
\end{array}\right\} C \frac{(2 \pi)^{1 / 2}}{(2 \lambda c)^{3 / 2}} \frac{2^{n+3} 2 n+1 !}{n ! \cos ^{2 n+2} \eta} \\
& \cdot\left[e^{\lambda c \sin \eta} \cos ^{4 n+3}(\pi / 4+\eta / 2) \pm e^{-\lambda c \sin \eta} \sin ^{4 n+3}(\pi / 4+\eta / 2)\right] \text {. }
\end{aligned}
$$

Les formules (42) et (43) peuvent évidemment être utilisées pour le calcul des valeurs numériques des fonctions $c e$ et $s e$ aux environs de $\eta=0$. A titre 
d'exemple, nous reprendrons le cas cité plus haut de $c e_{0}(0)$ pour $\lambda c=80$. La formule (42) donne, en ne conservant que le terme dominant dans (4)

$$
\begin{aligned}
c e_{0}(0) & =\frac{(2 \lambda c)^{1 / 2}}{(2 \pi)^{1 / 2}} e^{-\lambda c} \frac{(\pi \lambda c)^{1 / 4}}{(2)^{1 / 2}} \frac{(2 \pi)^{1 / 2}}{(2 \lambda c)^{1 / 2}} 2(2)^{1 / 2}=2(\pi \lambda c)^{1 / 4} e^{-\lambda c} \\
& =1,43724 \cdot 10^{-34} .
\end{aligned}
$$

Si l'on désire utiliser, pour plus d'exactitude, plusieurs termes du développement de Goldstein, on devra, pour déterminer le coefficient de raccordement, utiliser la valeur de $c e_{n}(0)$ ou de $s e_{n}^{\prime}(0)$ obtenue par la méthode indiquée plus haut. On constate, en procédant ainsi, que les deux développements asymptotiques (3) et (42), bien qu'ayant des domaines d'utilisation différents, donnent des valeurs concordantes dans une région assez étroite. C'est cette propriété qui a été utilisée par $\mathrm{G}$. Blanch pour calculer la valeur de $c e_{n}(0)$ pour $\lambda c=80$.

Pour représenter les fonctions de Mathieu aux environs de $\eta=\pi / 2$, on peut envisager également de développer, dans (38), la première exponentielle en série de puissances décroissantes de $\lambda c$ la de manière suivante

$$
e^{\lambda c \sin \eta \sin \eta_{0}}=e^{\lambda c-1 / 4\left(\alpha^{2}+\alpha_{0}^{2}\right)}\left[1+\frac{8 \alpha_{0}^{2}-\alpha^{4}+2 \alpha^{2} \alpha_{0}^{2}-\alpha_{0}^{4}}{32(\lambda c)^{2}}+\cdots\right]
$$

et utiliser, sous le signe intégrale, la représentation (3).

La calcul montre que l'on retrouve par cette méthode le même développement (3). On peut évidemment calculer ainsi $\bar{\lambda}_{n}$ et $\bar{\mu}_{n}$, mais le calcul est encore plus laborieux que par le procédé utilisé plus haut, et le résultat est identique.

\section{Fonctions SPHEROIDALES ALLONGÉES}

1. Les fonctions sphéroidales associées à l'ellipsoide de révolution allongé sont les solutions de l'équation différentielle

$$
\frac{d}{d \mu}\left[\left(1-\mu^{2}\right) \frac{d y}{d \mu}\right]+\left(a-\lambda^{2} c^{2} \mu^{2}-\frac{m^{2}}{1-\mu^{2}}\right) y=0
$$

telles que le produit $\left(1-\mu^{2}\right)^{-m / 2} y(\mu)$ est régulier aux environs des points $\mu= \pm 1$.

Dans ce qui suit, nous désignerons par $P e_{n}^{m}(\mu)$ celle de ces solutions qui se réduit à la fonction sphérique associée $P_{n}^{m}(\mu)$ lorsque $c=0$ et qui vérifie la condition de normalisation habituelle

$$
\int_{-1}^{1}\left[P e_{n}^{m}(\mu)\right]^{2} d \mu=\frac{2}{2 n+1} \frac{n-m !}{n+m !} .
$$

La valeur correspondante de la constante de séparation $a$ sera représentée par $a_{n}^{m}$. 
On sait que, lorsque $\lambda c$ est très grand, les fonctions $P e_{n}^{m}(\mu)$ peuvent être représentées asymptotiquement, aux environs de $\mu=0$, par la formule suivante

$$
\begin{aligned}
P e_{n}^{m}(\mu)= & C\left(1-\mu^{2}\right)^{m / 2}\left\{D_{p}(\alpha)+\frac{1}{2 \lambda c}\left[-\frac{1}{16} D_{p+4}(\alpha)+\frac{m}{2} D_{p+2}(\alpha)\right.\right. \\
& \left.+\frac{m p(p-1)}{2} D_{p-2}(\alpha)+\frac{p(p-1)(p-2)(p-3)}{16} D_{p-4}(\alpha)\right] \\
& +\frac{1}{(2 \lambda c)^{2}}\left[\frac{1}{512} D_{p+8}(\alpha)-\frac{m}{32} D_{p+6}(\alpha)-\frac{2 p+5-4 m^{2}}{32} D_{p+4}(\alpha)\right. \\
& -\frac{\left(p^{2}-25 p-36\right) m}{32} D_{p+2}(\alpha)+\frac{\left(p^{2}+27 p-10\right) p(p-1) m}{32} D_{p-2}(\alpha) \\
& +\frac{\left(2 p-3+4 m^{2}\right) p(p-1)(p-2)(p-3)}{32} D_{p-4}(\alpha) \\
& +\frac{p(p-1) \cdots(p-5) m}{32} D_{p-6}(\alpha) \\
& \left.\left.+\frac{p(p-1) \cdots(p-7)}{512} D_{p-8}(\alpha)\right]+O\left((\lambda c)^{-3}\right)\right\}
\end{aligned}
$$

où $\alpha=(2 \lambda c)^{1 / 2} \mu$ et où la constante $C$ a la valeur suivante

$$
C=\frac{1}{p !}\left(\frac{4 \lambda c}{\pi}\right)^{1 / 4}\left(\frac{p+2 m !}{2 p+2 m+1}\right)^{1 / 2}\left(c_{0}+\frac{c_{1}}{2 \lambda c}+\frac{c_{2}}{(2 \lambda c)^{2}}+\cdots\right)^{-1 / 2}
$$

avec $c_{0}=1, c_{1}=-m(p+1 / 2)$,

$$
\begin{aligned}
c_{2}= & \frac{(p+1)(p+2)(p+3)(p+4)+p(p-1)(p-2)(p-3)}{256} \\
& -3 m^{2} \frac{(p+1)(p+2)+p(p-1)}{4}+3 m(m-1) \frac{2 p^{2}+2 p+1}{2} .
\end{aligned}
$$

Le nombre $p$ est égal à $n-m$.

Nous avons donné ces formules, sous une forme un peu différente, dans [1].

La représentation asymptotique qui précède donne la valeur de la fonction $P e_{n}^{m}(\mu)$, lorsque $\lambda c$ est grand et $n$ et $m$ petits, avec une exactitude remarquable pour les valeurs de $\mu$ contenues dans un domaine assez étroit voisin de $\mu=0$, domaine où, en fait, la fonction prend seulement des valeurs numériques appréciables. Pour les autres valeurs de $\mu$, et en particulier aux environs de $\mu=1$, la formule (3) donne des valeurs totalement inexactes et qui n'ont même pas l'ordre de grandeur correct. 
Nous donnerons ci-dessous des formules utilisables dans cette dernière région.

2. Les fonctions $P e_{n}^{m}(\mu)$ vérifient deux groupes d'équations intégrales linéaires

a.

(5) $P e_{n}^{m}(\mu)=\lambda_{n}^{m} \int_{-1}^{1} e^{-i \lambda c \mu \mu_{0}}\left(1-\mu^{2}\right)^{m / 2}\left(1-\mu_{0}^{2}\right)^{m / 2} P e_{n}^{m}\left(\mu_{0}\right) d \mu_{0}$.

b.

(6) $P e_{n}^{m}(\mu)=\Lambda_{n}^{m} \int_{-1}^{1} I_{m}\left[\lambda c\left(1-\mu^{2}\right)^{1 / 2}\left(1-\mu_{0}^{2}\right)^{1 / 2}\right] P e_{n}^{m}\left(\mu_{0}\right) d \mu_{0} \quad(n-m$ pair $)$.

(7) $P e_{n}^{m}(\mu)=\Lambda_{n}^{m} \int_{-1}^{1} I_{m}\left[\lambda c\left(1-\mu^{2}\right)^{1 / 2}\left(1-\mu_{0}^{2}\right)^{1 / 2}\right] \mu \mu_{0} P e_{n}^{m}\left(\mu_{0}\right) d \mu_{0} \quad(n-m$ impair $)$.

De ces équations on déduit que, pour $n-m$ pair

$$
\begin{gathered}
P e_{n}^{m}(0)=\lambda_{n}^{m} \int_{-1}^{1}\left(1-\mu^{2}\right)^{m / 2} P e_{n}^{m}(\mu) d \mu, \\
\lim _{\mu=1}\left(1-\mu^{2}\right)^{-m / 2} P e_{n}^{m}(\mu)=\Lambda_{n}^{m} \frac{(\lambda c)^{m}}{2^{m} m !} \int_{-1}^{1}\left(1-\mu^{2}\right)^{m / 2} P e_{n}^{m}(\mu) d \mu
\end{gathered}
$$

et, par conséquent

$$
\lim _{\mu=1}\left(1-\mu^{2}\right)^{-m / 2} P e_{n}^{m}(\mu)=\frac{(\lambda c)^{m}}{2^{m} m !} \frac{\Lambda_{n}^{m}}{\lambda_{n}^{m}} P e_{n}^{m}(0) .
$$

Pour $n-m$ impair, on a

$$
\begin{gathered}
P e_{n}^{\prime m}(0)=-i \lambda c \lambda_{n}^{m} \int_{-1}^{1}\left(1-\mu^{2}\right)^{m / 2} \mu P e_{n}^{m}(\mu) d \mu, \\
\lim _{\mu=1}\left(1-\mu^{2}\right)^{-m / 2} P e_{n}^{m}(\mu)=\Lambda_{n}^{m} \frac{(\lambda c)^{m}}{2^{m} m !} \int_{-1}^{1}\left(1-\mu^{2}\right)^{m / 2} \mu P e_{n}^{m}(\mu) d \mu
\end{gathered}
$$

et, par conséquent

$$
\lim _{\mu=1}\left(1-\mu^{2}\right)^{-m / 2} P e_{n}^{m}(\mu)=i \frac{(\lambda c)^{m-1}}{2^{m} m !} \frac{\Lambda_{n}^{m}}{\lambda_{n}^{m}} P e_{n}^{\prime m}(0) .
$$

La détermination de la valeur asymptotique des premiers membres de (8) et (9) se ramène donc à la recherche de la représentation asymptotique des constantes caractéristiques $\lambda_{n}^{m}$ et $\Lambda_{n}^{m}$. 
3. Il résulte de la représentation asymptotique (3) que la fonction $P e_{n}^{m}(\mu)$ ne prend des valeurs appréciables que dans le voisinage de $\mu=0$. Dans ces conditions, nous pourrons, dans les équations intégrales (6) et (7), remplacer la fonction de Bessel par son développement asymptotique

$$
I_{m}(z)=\frac{e^{z}}{(2 \pi z)^{1 / 2}}\left[1+\frac{4 m^{2}-1}{8 z}+\frac{\left(4 m^{2}-1\right)\left(4 m^{2}-9\right)}{128 z^{2}}+\cdots\right] .
$$

En développant suivant les puissances décroissantes de $\lambda c$, on obtient

$$
I_{m}\left[\lambda c\left(1-\mu^{2}\right)^{1 / 2}\right]\left(1-\mu^{2}\right)^{m / 2}=(2 \pi \lambda c)^{-1 / 2} e^{\lambda c-\alpha^{2} / 4 \Sigma^{(m)}}(\alpha)
$$

où, comme plus haut,

$$
\alpha=(2 \lambda c)^{1 / 2} \mu
$$

et où on a posé

$$
\begin{aligned}
\Sigma^{(m)}(\alpha)= & 1-\frac{1}{2^{5} \lambda c}\left[2^{2}(2 m-1) \alpha^{2}+\alpha^{4}\right] \\
& +\frac{1}{2^{11}(\lambda c)^{2}}\left[2^{4}(2 m-1)(2 m-5) \alpha^{4}+2^{3}(2 m-3) \alpha^{6}+\alpha^{8}\right] \\
& +\frac{1}{3 \cdot 2^{16}(\lambda c)^{3}}\left[-2^{6}(2 m-1)(2 m-5)(2 m-9) \alpha^{6}\right. \\
& -\left(4 m^{2}-1\right)\left\{\frac{1}{2^{3} \lambda c}-\frac{1}{2^{8}(\lambda c)^{2}}\left[2^{2}(2 m-3) \alpha^{2}+\alpha^{4}\right]\right. \\
& \left.+\frac{1}{2^{14}(\lambda c)^{3}}\left[2^{4}(2 m-3)(2 m-7) \alpha^{4}+2^{3}(2 m-5) \alpha^{6}+\alpha^{8}\right]\right\} \\
& +\left(4 m^{2}-1\right)\left(4 m^{2}-9\right)\left\{\frac{1}{2^{7}(\lambda c)^{2}}-\frac{1}{2^{12}(\lambda c)^{3}}\left[2^{2}(2 m-5) \alpha^{2}+\alpha^{4}\right]\right\} \\
& -\left(4 m^{2}-1\right)\left(4 m^{2}-9\right)\left(4 m^{2}-25\right) \frac{1}{3 \cdot 2^{10}(\lambda c)^{3}} \cdot
\end{aligned}
$$

Supposons maintenant $n-m$ impair. Nous aurons

$$
\begin{aligned}
\int_{-1}^{1} I_{m}\left[\lambda c\left(1-\mu^{2}\right)^{1 / 2}\right] P e_{n}^{m}(\mu) d \mu & \\
= & \frac{C}{(2 \lambda c)^{1 / 2}} \int_{-(2 \lambda c)^{1 / 2}}^{(2 \lambda c)^{1 / 2}} I_{m}\left(\lambda c\left(1-\alpha^{2} / 2 \lambda c\right)^{1 / 2}\right)\left(1-\alpha^{2} / 2 \lambda c\right)^{m / 2} u_{n}^{m}(\alpha) d \alpha
\end{aligned}
$$


en appelant $u_{n}^{u}(\alpha)$ la quantité entre crochets dans (3). Pour $\lambda c$ très grand, ceci pourra encore s'écrire

$$
=C \frac{e^{\lambda c}}{2 \lambda c(\pi)^{1 / 2}} \int_{-\infty}^{\infty} e^{-\alpha^{2} / 2} \Sigma^{(m)}(\alpha) u_{n}^{m}(\alpha) d \alpha
$$

et par conséquent, en tenant compte de (6)

$$
\Lambda_{n}^{m}=\frac{(2)^{1 / 2} \lambda c e^{-\lambda c} u_{n}^{m}(0)}{\frac{1}{(2 \pi)^{1 / 2}} \int_{-\infty}^{\infty} e^{-\alpha^{2} / 4} \Sigma^{(m)}(\alpha) u_{n}^{m}(\alpha) d \alpha} .
$$

De même, pour $n-m$ impair, on aura

$$
\Lambda_{n}^{m}=\frac{2(2)^{1 / 2}(\lambda c)^{2} e^{-\lambda c} u_{n}^{\prime m}(0)}{\frac{1}{(2 \pi)^{1 / 2}} \int_{-\infty}^{\infty} e^{-\alpha^{2} / 4} \alpha \Sigma^{(m)}(\alpha) u_{n}^{m}(\alpha) d \alpha} .
$$

4. Le calcul effectif des valeurs asymptotiques de $\Lambda_{n}^{m}$ à partir de ces formules et de (3) est long et fastidieux. Nous donnerons ci-après les valeurs calculées pour les 10 premières fonctions $P e_{n}^{m}$. Il est à noter que, pour ce calcul, nous avons du, dans certains cas, tenir compte de termes en $(\lambda c)^{-\mathbf{3}}$ dans le développement (3).

a. $n-m$ pair.

$$
\begin{aligned}
& \Lambda_{0}^{0}=(2)^{1 / 2} \lambda c e^{-\lambda c}\left(1-\frac{1}{2^{2} \lambda c}-\frac{19}{2^{7}(\lambda c)^{2}}\right), \\
& \Lambda_{1}^{1}=(2)^{1 / 2} \lambda c e^{-\lambda c}\left(1+\frac{1}{2^{2} \lambda c}+\frac{15}{2^{7}(\lambda c)^{2}}\right), \\
& \Lambda_{2}^{2}=(2)^{1 / 2} \lambda c e^{-\lambda c}\left(1+\frac{7}{2^{2} \lambda c}+\frac{429}{2^{7}(\lambda c)^{2}}\right), \\
& \Lambda_{3}^{3}=(2)^{1 / 2} \lambda c e^{-\lambda c}\left(1+\frac{17}{2^{2} \lambda c}+\frac{1466}{2^{7}(\lambda c)^{2}}\right), \\
& \Lambda_{2}^{0}=8(2)^{1 / 2}(\lambda c)^{2} e^{-\lambda c}\left(1-\frac{6}{2^{2} \lambda c}-\frac{117}{2^{7}(\lambda c)^{2}}\right), \\
& \Lambda_{3}^{1}=8(2)^{1 / 2}(\lambda c)^{2} e^{-\lambda c}\left(1-\frac{20}{2^{2} \lambda c}+\frac{2461}{2^{7}(\lambda c)^{2}}\right) .
\end{aligned}
$$


b. $n-m$ impair.

$$
\begin{aligned}
& \Lambda_{1}^{0}=2(2)^{1 / 2}(\lambda c)^{2} e^{-\lambda c}\left(1-\frac{1}{2 \lambda c}-\frac{55}{2^{7}(\lambda c)^{2}}\right), \\
& \Lambda_{3}^{0}=\frac{16}{3}(2)^{1 / 2}(\lambda c)^{3} e^{-\lambda c}\left(1-\frac{9}{2^{2} \lambda c}-\frac{177}{2^{7}(\lambda c)^{2}}\right), \\
& \Lambda_{2}^{1}=2(2)^{1 / 2}(\lambda c)^{2} e^{-\lambda c}\left(1+\cdots+\frac{27}{2^{7}(\lambda c)^{2}}\right), \\
& \Lambda_{3}^{2}=2(2)^{1 / 2}(\lambda c)^{2} e^{-\lambda c}\left(1+\frac{3}{2 \lambda c}+\frac{729}{2^{7}(\lambda c)^{2}}\right) .
\end{aligned}
$$

Le calcul des 17 premières valeurs de $\Lambda_{n}^{m}$ nous a montré que, dans tous les cas, le terme dominant du développment asymptotique avait la forme suivante a. $n-m$ pair.

$$
\Lambda_{n}^{m}=2^{2 p+1 / 2}(\lambda c)^{p / 2+1} \frac{(p / 2) !}{p !} e^{-\lambda c} .
$$

b. $n-m$ impair.

$$
\Lambda_{n}^{m}=2^{2 p-1 / 2}(\lambda c)^{p / 2+3 / 2} \frac{((p-1) / 2) !}{p !} e^{-\lambda c} .
$$

Ces relations sont vraisemblablement exactes dans tous les cas, mais nous n'avons pas pu le démontrer jusqu'ici.

5. Nous allons maintenant calculer la valeur asymptotique des constantes caractéristiques $\lambda_{n}^{m}$ de l'équation intégrale (5). Ce calcul est beaucoup moins compliqué que celui des $\Lambda_{n}^{m}$. On remplace, dans l'équation, $\mu$ par $(2 \lambda c)^{-1 / 2} \alpha$ et $P e_{n}^{m}$ par son développement asymptotique (3), puis on développe en série de puissances décroissantes de $\lambda c$. En prenant comme limites d'intégration $-\infty$ et $\infty$ au lieu de $\pm(2 \lambda c)^{1 / 2}$ et en tenant compte de ce que

$$
\int_{-\infty}^{\infty} e^{-i \alpha \alpha_{0} / 2} D_{p}\left(\alpha_{0}\right) d \alpha_{0}=2 i^{-p}(\pi)^{1 / 2} D_{p}(\alpha)
$$

on constate que le second membre peut se mettre sous forme d'une série de fonctions paraboliques. En identifiant les coefficients de $D_{p}(\alpha)$ dans les deux membres, on trouve que

$$
\begin{aligned}
\lambda_{n}^{m}=i^{p} \frac{(2 \lambda c)^{1 / 2}}{2(\pi)^{1 / 2}}[1 & -\frac{m(2 p+1)}{2 \lambda c} \\
& \left.+\frac{m^{2}\left(4 p^{2}+4 p+1\right)-3 m\left(2 p^{2}+2 p+1\right)}{8(\lambda c)^{2}}+\cdots\right]^{-1} .
\end{aligned}
$$


Le calcul est en tous points identique à celui des constantes caractéristiques de l'équation intégrale (I, 5) des fonctions de Mathieu.

6 . Connaissant ainsi la valeur des constantes $\Lambda_{n}^{m}$ et $\lambda_{n}^{m}$, il est maintenant possible de calculer

$$
\lim _{\mu=1}\left(1-\mu^{2}\right)^{-m / 2} P e_{n}^{m}(\mu)
$$

à partir de (10) et (13).

Pour vérifier numériquement l'exactitude de nos formules, nous avons comparé les valeurs numériques qu'elles fournissent avec celles obtenues au moyen des tables de J. A. Stratton et al [6] pour la valeur de $\lambda c$ la plus élevée contenue dans ces tables, c'est-à-dire $\lambda c=8$.

Pour cette comparaison, nous avons tenu compte de ce que la condition de normalisation de ces tables est différente de la normalisation rationnelle généralement admise et exprimée par (2). Le tableau ci-dessous contient les résultats obtenus. On voit que, comme toujours avec ce genre de développements, l'exactitude est très bonne pour $n$ et $m$ petits, mais diminue progressivement au fur et à mesure que $n$ et $m$ augmentent.

\begin{tabular}{l|c|c|c|c|c|c}
\hline \multicolumn{1}{c|}{$n=$} & 0 & 1 & 2 & 1 & 2 & 2 \\
\hline \multicolumn{1}{c|}{$m=$} & 0 & 0 & 0 & 1 & 1 & 2 \\
\hline$P e_{n}^{m}(0)$ exact & 1,7628 & 0 & $-0,5195$ & 1,4434 & 0 & 3,9051 \\
$P e_{n}^{m}(0)$ approximatif & 1,7634 & 0 & $-0,5246$ & 1,4192 & 0 & 3,7640 \\
$P e_{n}^{\prime m}(0)$ exact & 0 & 3,8451 & 0 & 0 & 7,3873 & 0 \\
$P e_{n}^{\prime m}(0)$ approximatif & 0 & 3,8573 & 0 & 0 & 7,3052 & 0 \\
$\lim _{\mu=1} P e_{n}^{m}(\mu)$ exact & 0,005726 & 0,02400 & 0,08790 & 0,01881 & 0,1604 & 0,1179 \\
\hline
\end{tabular}

7. Ayant ainsi obtenu la représentation asymptotique de

$$
\lim _{\mu=1}\left(1-\mu^{2}\right)^{-m / 2} P e_{n}^{m}(\mu)
$$

il nous faut maintenant rechercher la valeur de la fonction $P e_{n}^{m}(\mu)$ elle-même aux environs de $\mu=1$.

Nous utiliserons pour cela les équations intégrales (6) et (7) où, dans le second membre, nous remplacerons la fonction $P e_{n}^{m}(\mu)$ par sa valeur asymptotique (3). Il est bien évident que le calcul se ramène à celui des intégrales définies suivantes 


$$
\begin{aligned}
I_{2 p}^{m} & =\int_{-1}^{1} I_{m}\left[\lambda c\left(1-\mu^{2}\right)^{1 / 2}\left(1-\mu_{0}^{2}\right)^{1 / 2}\right] e^{-\lambda c \mu_{0}^{2} / 2} H e_{2 p}\left((2 \lambda c)^{1 / 2} \mu_{0}\right) d \mu_{0}, \\
I_{2 p+1}^{m} & =\int_{-1}^{1} I_{m}\left[\lambda c\left(1-\mu^{2}\right)^{1 / 2}\left(1-\mu_{0}^{2}\right)^{1 / 2}\right] \mu \mu_{0} e^{-\lambda c \mu_{0}^{2} / 2} H e_{2 p+1}\left((2 \lambda c)^{1 / 2} \mu_{0}\right) d \mu_{0} .
\end{aligned}
$$

La fonction $I_{m}(z)$ est une fonction rapidement croissante de $z$ pour les valeurs pas trop faibles de $z$ et pas trop grandes de $m$, ce qui est le cas actuellement, mais on sait que si l'on pose

$$
I_{m}(z)=e^{z} \phi_{m}(z)
$$

la fonction $\phi_{m}(z)$ ne varie que très lentement avec $z$. En posant

$$
\mu_{1}=\left(1-\mu^{2}\right)^{1 / 2}
$$

nous pourrons donc écrire, en remplaçant dans l'exponentielle $\left(1-\mu_{0}^{2}\right)^{1 / 2}$ par $1-\mu_{0}^{2} / 2$

$$
\begin{aligned}
I_{2 p}^{m}= & e^{\lambda c \mu_{1}} \int_{-\infty}^{\infty} e^{-\lambda c\left(1+\mu_{1}\right) \mu_{0}{ }^{2} / 2}\left[\phi_{m}\left(\lambda c \mu_{1}\right)-\frac{\lambda c \mu_{1}}{2} \mu_{0}^{2} \phi_{m}^{\prime}\left(\lambda c \mu_{1}\right)\right. \\
& \left.+\frac{\left(\lambda c \mu_{1}\right)^{2}}{8} \mu_{0}^{4} \phi_{m}^{\prime \prime}\left(\lambda c \mu_{1}\right)-\cdots\right] H e_{2 p}\left((2 \lambda c)^{1 / 2} \mu_{0}\right) d \mu_{0}
\end{aligned}
$$

ou encore, en posant en outre

$$
\begin{aligned}
& \left(\lambda c\left(1+\mu_{1}\right)\right)^{1 / 2} \mu_{0}=t, \\
I_{2 p}^{m}=\frac{e^{\lambda c \mu_{1}}}{\left(\lambda c\left(1+\mu_{1}\right)\right)^{1 / 2}} \int_{-\infty}^{\infty} e^{-t^{2} / 2} & {\left[\phi_{m}-\frac{\mu_{1} t^{2}}{2\left(1+\mu_{1}\right)} \phi_{m}^{\prime}\right.} \\
& \left.\quad+\frac{\mu_{1}^{2} t^{4}}{8\left(1+\mu_{1}\right)^{2}}-\cdots\right] H e_{2 p}\left(t\left(\frac{1+\mu_{1}}{2}\right)^{-1 / 2}\right) d t .
\end{aligned}
$$

En tenant compte des intégrales définies suivantes

$$
\begin{aligned}
\int_{-\infty}^{\infty} e^{-t^{2} / 2} H e_{2 p}(\alpha t) d t & =(2 \pi)^{1 / 2} \frac{2 p !}{2^{p} p !}\left(\alpha^{2}-1\right)^{p} \\
\int_{-\infty}^{\infty} e^{-t^{2} / 2} t^{2} H e_{2 p}(\alpha t) d t & =(2 \pi)^{1 / 2} \frac{2 p !}{2^{p} p !}\left[(2 p+1)\left(\alpha^{2}-1\right)^{p}+2 p\left(\alpha^{2}-1\right)^{p-1}\right] \\
\int_{-\infty}^{\infty} e^{-t^{2} / 2} t^{4} H e_{2 p}(\alpha t) d t= & (2 \pi)^{1 / 2} \frac{2 p !}{2^{p} p !}\left[(2 p+1)(2 p+3)\left(\alpha^{2}-1\right)^{p}\right. \\
& \left.+4 p(2 p+1)\left(\alpha^{2}-1\right)^{p-1}+4 p(p-1)\left(\alpha^{2}-1\right)^{p-2}\right]
\end{aligned}
$$

il vient 


$$
\begin{aligned}
I_{2 p}^{m}= & \frac{e^{\lambda c \mu_{1}}}{\left(\lambda c\left(1+\mu_{1}\right)\right)^{1 / 2}}(2 \pi)^{1 / 2} \frac{2 p !}{2^{p} p !}\left[\phi_{m}\left(\frac{1-\mu_{1}}{1+\mu_{1}}\right)^{p}\right. \\
& -\frac{\mu_{1} \phi_{m}^{\prime}}{2\left(1+\mu_{1}\right)}\left((2 p+1)\left(\frac{1-\mu_{1}}{1+\mu_{1}}\right)^{p}+2 p\left(\frac{1-\mu_{1}}{1+\mu_{1}}\right)^{p-1}\right. \\
& +\frac{\mu_{1}^{2} \phi_{m}^{\prime \prime}}{8\left(1+\mu_{1}\right)^{2}}\left((2 p+1)(2 p+3)\left(\frac{1-\mu_{1}}{1+\mu_{1}}\right)^{p}\right. \\
& \left.\left.+4 p(2 p+1)\left(\frac{1-\mu_{1}}{1+\mu_{1}}\right)^{p-1}+4 p(p-1)\left(\frac{1-\mu_{1}}{1+\mu_{1}}\right)^{p-2}\right)-\cdots\right] .
\end{aligned}
$$

Comme, d'autre part

$$
\begin{aligned}
& e^{z} \phi_{m}(z)=I_{m}(z) \\
& e^{z} \phi_{m}^{\prime}(z)=2^{-1}\left(I_{m+1}(z)-2 I_{m}(z)+I_{m-1}(z)\right) \\
& e^{z} \phi_{m}^{\prime \prime}(z)=-4^{-1}\left(I_{m+2}(z)-4 I_{m+1}(z)+6 I_{m}(z)-4 I_{m-1}(z)+I_{m-2}(z)\right)
\end{aligned}
$$

on a donc finalement l'expression asymptotique désirée qui ne contient que des fonctions parfaitement connues.

En faisant tendre $\mu_{1}$ vers 0 , on trouve

$$
\begin{aligned}
& \lim _{\mu_{1}=0} \mu_{1}^{-m} I_{2 p}^{m}(\mu)=\frac{(2 \pi)^{1 / 2}}{(\lambda c)^{1 / 2}} \\
& \cdot \frac{2 p !(\lambda c)^{m}}{2^{p} p ! 2^{m} m !}\left[1-\frac{(4 p+1) m}{4 \lambda c}-\frac{\left(16 p^{2}+8 p+3\right) m(m-1)}{8(\lambda c)^{2}}+\cdots\right] .
\end{aligned}
$$

La même valeur s'obtient directement en faisant tendre $1-\mu^{2}$ vers 0 dans (24), ce qui montre que les expressions asymptotiques que nous avons obtenues pour $I_{p}^{m}$ sont utilisables jusque $\mu=1$.

Dans les cas particuliers où $m=0, p=0$, ou 2 , on trouve

$$
\begin{aligned}
I_{0}^{0}(\mu)=\frac{(2 \pi)^{1 / 2}}{\left(\lambda c\left(1+\mu_{1}\right)\right)^{1 / 2}}\left[I_{0}+\frac{\mu_{1}\left(I_{0}-I_{1}\right)}{2\left(1+\mu_{1}\right)}-\frac{3 \mu_{1}^{2}\left(3 I_{0}-4 I_{1}+I_{2}\right)}{16\left(1+\mu_{1}\right)^{2}}+\cdots\right], \\
I_{4}^{0}(\mu)=\frac{3(2 \pi)^{1 / 2}}{\left(\lambda c\left(1+\mu_{1}\right)\right)^{1 / 2}}\left[\left(\frac{1-\mu_{1}}{1+\mu_{1}}\right)^{2} I_{0}\right. \\
\left.\quad+\frac{\mu_{1}\left(I_{0}-I_{1}\right)}{2\left(1+\mu_{1}\right)}\left(5\left(\frac{1-\mu_{1}}{1+\mu_{1}}\right)^{2}+4\left(\frac{1-\mu_{1}}{1+\mu_{1}}\right)\right)+\cdots\right] .
\end{aligned}
$$

Un calcul en tous points semblable donne 


$$
\begin{aligned}
I_{2 p+1}^{m}(\mu) & =\frac{2 \mu e^{\lambda c \mu_{1}}}{\lambda c\left(1+\mu_{1}\right)^{3 / 2}}(\pi)^{1 / 2} \frac{2 p+1 !}{2^{p} p !}\left[\phi_{m}\left(\frac{1-\mu_{1}}{1+\mu_{1}}\right)^{p}\right. \\
& -\frac{\mu_{1} \phi_{m}^{\prime}}{2\left(1+\mu_{1}\right)}\left((2 p+3)\left(\frac{1-\mu_{1}}{1+\mu_{1}}\right)^{p}+2 p\left(\frac{1-\mu_{1}}{1+\mu_{1}}\right)^{p-1}\right) \\
& +\frac{\mu_{1}^{2} \phi_{m}^{\prime \prime}}{8\left(1+\mu_{1}\right)^{2}}\left((2 p+3)(2 p+5)\left(\frac{1-\mu_{1}}{1+\mu_{1}}\right)^{p}\right. \\
& \left.\left.+4 p(2 p+3)\left(\frac{1-\mu_{1}}{1+\mu_{1}}\right)^{p-1}+4 p(p-1)\left(\frac{1-\mu_{1}}{1+\mu_{1}}\right)^{p-2}\right)+\cdots\right]
\end{aligned}
$$

et

$$
\begin{aligned}
\lim _{\mu_{1}=0} \mu_{1}^{-m} I_{2 p+1}^{m}(\mu)= & \frac{2(\pi)^{1 / 2} 2 p+1 !(\lambda c)^{m}}{\lambda c 2^{p} p ! 2^{m} m !} \\
& \cdot\left[1-\frac{(4 p+3) m}{4 \lambda c}-\frac{\left(16 p^{2}+24 p+15\right) m(m-1)}{8(\lambda c)^{2}} \ldots\right] .
\end{aligned}
$$

Dans les cas particuliers où $m=0, p=0$ ou 2 , on trouve

$$
\begin{aligned}
I_{1}^{0}=\frac{2 \mu(\pi)^{1 / 2}}{\lambda c\left(1+\mu_{1}\right)^{3 / 2}} & {\left[I_{0}+\frac{3 \mu_{1}\left(I_{0}-I_{1}\right)}{2\left(1+\mu_{1}\right)}+\frac{15 \mu_{1}^{2}\left(3 I_{0}-4 I_{1}+I_{2}\right)}{16\left(1+\mu_{1}\right)^{2}}-\cdots\right], } \\
I_{5}^{0}=\frac{30 \mu(\pi)^{1 / 2}}{\lambda c\left(1+\mu_{1}\right)^{3 / 2}} & {\left[I_{0}\left(\frac{1-\mu_{1}}{1+\mu_{1}}\right)^{2}\right.} \\
& \left.+\frac{\mu_{1}\left(I_{0}-I_{1}\right)}{2\left(1+\mu_{1}\right)}\left(7\left(\frac{1-\mu_{1}}{1+\mu_{1}}\right)^{2}+4\left(\frac{1-\mu_{1}}{1+\mu_{1}}\right)\right)+\cdots\right] .
\end{aligned}
$$

Connaissant $I_{p}^{m}$, on en tirera maintenant facilement la valeur asymptotique de $P e_{n}^{m}(\mu)$. On a en effet

$$
\begin{aligned}
P e_{n}^{m}(\mu)=C \Lambda_{n}^{m}\left[I_{p}^{m}\right. & +\frac{1}{2 \lambda c}\left(-\frac{I_{p+4}^{m}}{16}+\frac{m I_{p+2}^{m}}{2}+\frac{m p(p-1) I_{p-2}^{m}}{2}\right. \\
& \left.\left.+\frac{p(p-1)(p-2)(p-3)}{16} I_{p-4}^{m}\right)+\frac{1}{(2 \lambda c)^{2}}(\cdots)+\cdots\right] .
\end{aligned}
$$

Lorsque le produit $\lambda c \mu$ est assez grand, on a approximativement

$$
\begin{array}{rlrl}
P e_{n}^{m}(\mu) & =C \Lambda_{n}^{m} \frac{(2 \pi)^{1 / 2}}{\left(\lambda c\left(1+\mu_{1}\right)\right)^{1 / 2}} \cdot \frac{2 p !}{2^{p} p !}\left(\frac{1-\mu_{1}}{1+\mu_{1}}\right)^{p} I_{m}\left(\lambda c \mu_{1}\right) & (n-m=2 p) \\
& =C \Lambda_{n}^{m} \frac{2 \mu(\pi)^{1 / 2}}{\lambda c\left(1+\mu_{1}\right)^{3 / 2}} \cdot \frac{2 p+1 !}{2^{p} p !}\left(\frac{1-\mu_{1}}{1+\mu_{1}}\right)^{p} I_{m}\left(\lambda c \mu_{1}\right) \quad(n-m=2 p+1) .
\end{array}
$$


En prenant pour $\Lambda_{n}^{m}$ et $C$ également les termes dominants des développements asymptotiques, (en admettant que (21) et (22) sont exacts dans tous les cas), on trouve finalement

$$
\begin{array}{r}
P e_{n}^{m}(\mu)=\frac{1}{2 p !}\left(\frac{n+m !}{2 n+1}\right)^{1 / 2}\left(\frac{\pi}{\lambda c}\right)^{1 / 4} 2^{3 p+3 / 2}(\lambda c)^{p+1} e^{-\lambda c} \frac{\left(1-\mu_{1}\right)^{p}}{\left(1+\mu_{1}\right)^{p+1 / 2}} I_{m}\left(\lambda c \mu_{1}\right), \\
(n-m=2 p)
\end{array}
$$

$$
\begin{array}{r}
P e_{n}^{m}(\mu)=\frac{1}{2 p+1 !}\left(\frac{n+m !}{2 n+1}\right)^{1 / 2}(4 \pi \lambda c)^{1 / 4} 2^{3 p+5 / 2}(\lambda c)^{p+1} e^{-\lambda c} \frac{\mu\left(1-\mu_{1}\right)^{p}}{\left(1+\mu_{1}\right)^{p+3 / 2}} I_{m}\left(\lambda c \mu_{1}\right) \\
(n-m=2 p+1) .
\end{array}
$$

Dans le cas particulier où $p=m=0$, ceci devient

$$
\begin{aligned}
& P e_{0}^{0}(\mu)=2(2)^{1 / 2} \pi^{1 / 4}(\lambda c)^{3 / 4} e^{-\lambda c}\left(1+\mu_{1}\right)^{-1 / 2} I_{0}\left(\lambda c \mu_{1}\right), \\
& P e_{1}^{0}(\mu)=8(3)^{-1 / 2} \pi^{1 / 4}(\lambda c)^{5 / 4} e^{-\lambda c}\left(1+\mu_{1}\right)^{-3 / 2} \mu I_{0}\left(\lambda c \mu_{1}\right) .
\end{aligned}
$$

Comme vérification numérique de ces formules, nous y ferons $\mu_{1}=0, \mu=1$, $\lambda c=8$, ce qui donne

$$
\begin{array}{ll}
P e_{0}^{0}(1)=\pi^{1 / 4} 2^{15 / 4} e^{-8}=0,006004 & (\text { exact: } 0,005726), \\
P e_{1}^{0}(1)=\pi^{1 / 4} 8^{9 / 4} 3^{-1 / 2} e^{-8}=0,02775 & \text { (exact: } 0,02400),
\end{array}
$$

Etant donné qu'on n'a tenu compte que du terme dominant du développement asymptotique et que la valeur $\lambda c=8$ n'est pas particulièrement élevée, l'exactitude obtenue peut être considérée comme très bonne.

Pour le calcul effectif de la fonction $P e_{n}^{m}(\mu)$, il y a avantage à utiliser la relation suivante, qui se déduit immédiatement de (31), (33), (34), et (35)

$$
\frac{P e_{n}^{m}(\mu)}{\lim _{\mu=1}\left(1-\mu^{2}\right)^{-m / 2} P e_{n}^{m}(\mu)}
$$

$$
=\frac{I_{p}^{m}\left(\mu_{1}\right)+\frac{1}{2 \lambda c}\left(-\frac{I_{p+4}^{m}\left(\mu_{1}\right)}{16}+\cdots\right)+\cdots}{\lim _{\mu_{1}=0} \mu_{1}^{-m}\left[I_{n}^{m}\left(\mu_{1}\right)+\frac{1}{2 \lambda c}\left(-\frac{I_{p+4}^{m}\left(\mu_{1}\right)}{16}+\cdots\right)+\cdots\right]}
$$

et à utiliser la valeur du dénominateur du premier membre calculée comme indiqué plus haut, (10) et (13).

On trouve ainsi, en particulier, pour $m=0, n=0$ et 1 


$$
\begin{aligned}
& \frac{P e_{0}^{0}(\mu)}{P e_{0}^{0}(1)}=\left(\frac{\lambda c}{2 \pi}\right)^{1 / 2}\left(1-\frac{3}{32 \lambda c}\right)^{-1}\left[I_{0}^{0}\left(\lambda c \mu_{1}\right)-\frac{1}{32 \lambda c} I_{4}^{0}\left(\lambda c \mu_{1}\right)\right], \\
& \frac{P e_{1}^{0}(\mu)}{P e_{1}^{0}(1)}=\left(\frac{\lambda c}{2 \pi}\right)^{1 / 2}\left(1-\frac{15}{32 \lambda c}\right)^{-1}\left[I_{0}^{1}\left(\lambda c \mu_{1}\right)-\frac{1}{32 \lambda c} I_{5}^{0}\left(\lambda c \mu_{1}\right)\right]
\end{aligned}
$$

où on utilisera pour $I_{0}^{0}, I_{4}^{0}, I_{1}^{0}$ et $I_{5}^{0}$ les valeurs (33.1), (33.2), (35.1) et (35.2).

A titre d'exemple numérique, nous avons comparé les valeurs obtenues ainsi pour $\mu=0,9, \lambda c=8$ avec les valeurs exactes fournies par les tables de J. A. Stratton et al. On trouve, tous calculs faits

$$
\begin{aligned}
& \left.\frac{P e_{0}^{0}(0,9)}{P e_{0}^{0}(1)}=6,2853 \quad \text { (Valeur exacte: } 6,2643\right), \\
& \left.\frac{P e_{1}^{0}(0,9)}{P e_{1}^{0}(1)}=4,1640 \quad \text { (Valeur exacte: } 4,1825\right) .
\end{aligned}
$$

On voit que l'erreur ne dépasse pas $0,5 \%$ environ, bien que, comme nous l'avons déja dit, la valeur $\lambda c=8$ ne soit pas spécialement élevée.

\section{Fonctions Sphéroidales aplaties}

1. Les fonctions sphéroidales associées à l'ellipsoide de révolution aplati sont les solutions de l'équation différentielle

$$
\frac{d}{d \mu}\left[\left(1-\mu^{2}\right) \frac{d y}{d \mu}\right]+\left(a+\lambda^{2} c^{2} \mu^{2}-\frac{m^{2}}{1-\mu^{2}}\right) y=0
$$

telles que le produit

$$
\left(1-\mu^{2}\right)^{-m / 2} y(\mu)
$$

est régulier aux environs de $\mu= \pm 1$.

Dans ce qui suit, nous désignerons par $\bar{P} e_{n}^{m}(\mu)$ la solution de (1) qui se réduit à la fonction sphérique associée $P_{n}^{m}(\mu)$ pour $\lambda c=0$ et qui vérifie la condition de normalisation habituelle

$$
\int_{-1}^{1}\left[\bar{P} e_{n}^{m}(\mu)\right]^{2} d \mu=\frac{2}{2 n+1} \cdot \frac{n-m !}{n+m !} .
$$

La valeur correspondante de la constante de séparation sera représentée $\operatorname{par} b_{n}^{m}$.

On sait que lorsque $\lambda c$ est très grand, les fonctions $\bar{P} e_{n}^{m}(\mu)$ peuvent être représentées asymptotiquement, aux environs de $\mu=1$, par la formule suivante 


$$
\begin{aligned}
& P e_{n}^{m}(\mu)=C t^{m / 2} e^{-t / 2}\left\{L_{p}^{(m)}(t)+\frac{1}{2 \lambda c}\left[-\frac{(p+1)(p+2)}{4} L_{p+2}^{(m)}(t)\right.\right. \\
& \left.+\frac{p+1}{2} L_{p+1}^{(m)}(t)+\frac{p+m}{2} L_{p-1}^{(m)}(t)+\frac{(p+m)(p+m-1)}{4} L_{p-2}^{(m)}(t)\right] \\
& +\frac{1}{(2 \lambda c)^{2}}\left[\frac{(p+1)(p+2)(p+3)(p+4)}{32} L_{p+4}^{(m)}(t)\right. \\
& -\frac{(p+1)(p+2)(3 p+5)}{24} L_{p+3}^{(m)}(t)-\frac{(p+1)(p+2)(4 p+2 m+5)}{8} L_{p+2}^{(m)}(t) \\
& (3)-\frac{(p+1)\left(p^{2}+p m-12 p-6 m-12\right)}{8} L_{p+1}^{(m)}(t) \\
& +\frac{(p+m)\left(p^{2}+p m+14 p+7 m+1\right)}{8} L_{p-1}^{(m)}(t) \\
& +\frac{(p+m-1)(p+m)(p+m+1)}{4} L_{p-2}^{(m)}(t) \\
& +\frac{(p+m)(p+m-1)(p+m-2)}{8} L_{p-3}^{(m)}(t) \\
& \left.\left.+\frac{(p+m)(p+m-1)(p+m-2)(p+m-3)}{32} L_{p-4}^{(m)}(t)\right]+O\left(\lambda c^{-3}\right)\right\}
\end{aligned}
$$

où $t=\lambda c\left(1-\mu^{2}\right)$ et où la constante $C$ a la valeur suivante

(4) $C=2^{(m+1) / 2}\left(\frac{(\lambda c)^{m+1}}{2 n+1} \frac{n+m ! p !}{n-m ! p+m !}\right)^{1 / 2}\left(c_{0}+\frac{c_{1}}{2 \lambda c}+\frac{c_{2}}{(2 \lambda c)^{2}}+\cdots\right)^{-1 / 2}$

avec $c_{0}=1, c_{1}=m+2 p+1$,

$$
\begin{aligned}
c_{2}= & \frac{(p+1)(p+2)(p+m+1)(p+m+2)}{16}+\frac{3(p+1)(p+m+1)}{4} \\
& +\frac{3 p(p+m)}{4}+\frac{p(p-1)(p+m)(p+m-1)}{16}+\frac{3(2 p+m+1)^{2}}{2} .
\end{aligned}
$$

Le nombre $p$ est défini par $n-m=2 p$ ou $n-m=2 p+1$ suivant que $n-m$ est pair ou impair.

Nous avons donné ces formules, sous une forme un peu différente, dans [1]. Il est à noter que la valeur de $c_{2}$, dans ce travail, est inexacte.

La représentation asymptotique qui précède donne la valeur de la fonction $\bar{P} e_{n}^{m}(\mu)$, lorsque $\lambda c$ est grand et $n$ et $m$ petits, avec une exactitude remarquable pour les valeurs de $\mu$ contenues dans un domaine assez étroit 
voisin de $\mu=1$, domaine où, en fait, la fonction prend seulement des valeurs apréciables. Pour les autres valeurs de $\mu$, la représentation asymptotique ci-dessus donne des valeurs totalement inexactes et qui n'ont même pas l'ordre de grandeur correct.

Nous donnerons ci-après, des formules utilisables dans cette dernière région.

2. Les fonctions sphéroidales aplaties vérifient deux groupes d'équations intégrales linéaires

a.

(5) $\bar{P} e_{n}^{m}(\mu)=\bar{\lambda}_{n}^{m} \int_{-1}^{1} e^{\lambda c \mu \mu_{0}}\left(1-\mu^{2}\right)^{m / 2}\left(1-\mu_{0}^{2}\right)^{m / 2} \bar{P} e_{n}^{m}\left(\mu_{0}\right) d \mu_{0}$.

b.

(6) $\bar{P} e_{n}^{m}(\mu)=\bar{\Lambda}_{n}^{m} \int_{-1}^{1} J_{m}\left[\lambda c\left(1-\mu^{2}\right)^{1 / 2}\left(1-\mu_{0}^{2}\right)^{1 / 2}\right] \bar{P} e_{n}^{m}\left(\mu_{0}\right) d_{\mu_{0}} \quad(n-m$ pair $)$,

(7) $\bar{P} e_{n}^{m}(\mu)=\bar{\Lambda}_{n}^{m} \int_{-1}^{1} J_{m}\left[\lambda c\left(1-\mu^{2}\right)^{1 / 2}\left(1-\mu_{0}^{2}\right)^{1 / 2}\right] \mu \mu_{0} \bar{P} e_{n}^{m}\left(\mu_{0}\right) d \mu_{0}(n-m$ impair $)$.

De ces équations on déduit que, pour $n-m$ pair

$$
\bar{P} e_{n}^{m}(0)=\bar{\lambda}_{n}^{m} \int_{-1}^{1}\left(1-\mu^{2}\right)^{m / 2} \bar{P} e_{n}^{m}(\mu) d \mu
$$

et

$$
\lim _{\mu=1}\left(1-\mu^{2}\right)^{-m / 2} \bar{P}_{n}^{m}(\mu)=\bar{\Lambda}_{n}^{m} \frac{(\lambda c)^{m}}{2^{m} m !} \int_{-1}^{1}\left(1-\mu^{2}\right)^{m / 2} \bar{P} e_{n}^{m}(\mu) d \mu
$$

d'où

$$
\frac{\bar{P} e_{n}^{m}(0)}{\lim _{\mu=1}\left(1-\mu^{2}\right)^{-m / 2} \bar{P} e_{n}^{m}(\mu)}=\frac{\bar{\lambda}_{n}^{m}}{\frac{(\lambda c)^{m}}{2^{m} m !} \bar{\Lambda}_{n}^{m}}
$$

tandis que pour $n-m$ impair

$$
\begin{gathered}
\bar{P} e_{n}^{\prime m}(0)=\lambda c \bar{\lambda}_{n}^{m} \int_{-1}^{1}\left(1-\mu^{2}\right)^{m / 2} \mu \bar{P} e_{n}^{m}(\mu) d \mu \\
\lim _{\mu=1}\left(1-\mu^{2}\right)^{-m / 2} \bar{P}_{n}^{m}(\mu)=\bar{\Lambda}_{n}^{m} \frac{(\lambda c)^{m}}{2^{m} m !} \int_{-1}^{1}\left(1-\mu^{2}\right)^{m / 2} \mu \bar{P}^{m} e_{n}^{m}(\mu) d \mu
\end{gathered}
$$

d'où 


$$
\frac{\bar{P}_{n}^{\prime m}(0)}{\lim \left(1-\mu^{2}\right)^{-m / 2} \bar{P} e_{n}^{m}(\mu)}=\frac{\lambda c \bar{\lambda}_{n}^{m}}{\frac{(\lambda c)^{m}}{2^{m} m !} \bar{\Lambda}_{n}^{m}}
$$

La détermination de la valeur asymptotique de $P e_{n}^{m}(0)$ et $P e_{n}^{\prime m}(0)$ se ramène donc à celle des constantes caractéristiques $\bar{\lambda}_{n}^{m}$ et $\bar{\Lambda}_{n}^{m}$.

3. Si l'on pose

$$
\bar{P} e_{n}^{m}(\mu)=e^{-t / 2} t^{m / 2} y(t)
$$

on aura évidemment

$$
\lim _{\mu=1}\left(1-\mu^{2}\right)^{-m / 2} \bar{P} e_{n}^{m}(\mu)=(\lambda c)^{m / 2} y(0)
$$

et le développement asymptotique de $y(0)$ se déduit immédiatement de (3).

Considérons maintenant l'équation intégrale (5) et supposons $\lambda c$ très grand et positif. Alors, seule la partie de l'intégrale prise entre 0 et 1 aura une valeur appréciable. En prenant $t$ comme variable au lieu de $\mu$, on pourra écrire

$$
\begin{aligned}
y(0) & =\frac{\bar{\lambda}_{n}^{m}}{2(\lambda c)^{m+1}} \int_{0}^{\lambda c} e^{\lambda c(1-t / \lambda c)^{1 / 2}-t / 2} t^{m}(1-t / \lambda c)^{-1 / 2} y(t) d t \\
& \sim \frac{e^{\lambda c} \bar{\lambda}_{n}^{m}}{2(\lambda c)^{m+1}} \int_{0}^{\infty} e^{-t} t^{m} \Sigma(t) y(t) d t
\end{aligned}
$$

en posant

$$
\Sigma(t)=(1-t / \lambda c)^{-1 / 2} e^{\lambda c(1-t / \lambda c)^{1 / 2}-\lambda c+t / 2} .
$$

En développant suivant les puissances décroissantes de $\lambda c$, on trouve ensuite

$$
\begin{aligned}
\Sigma(t)=1 & +\frac{1}{\lambda c}\left(\frac{t}{2}-\frac{t^{2}}{2^{3}}\right)+\frac{1}{(\lambda c)^{2}}\left(\frac{3 t^{2}}{2^{3}}-\frac{t^{3}}{2^{3}}+\frac{t^{4}}{2^{7}}\right) \\
& +\frac{1}{(\lambda c)^{3}}\left(\frac{5 t^{3}}{2^{4}}-\frac{15 t^{4}}{2^{7}}+\frac{3 t^{5}}{2^{8}}-\frac{t^{6}}{3 \cdot 2^{10}}\right)+\cdots
\end{aligned}
$$

En introduisant la valeur (3) de $y(t)$ et le développement (18) de $\Sigma(t)$ dans la relation (16), on peut en déduire la représentation asymptotique de $\bar{\lambda}_{n}^{m}$. Un calcul assez fastidieux conduit aux expressions suivantes $p=0$

$$
\bar{\lambda}_{n}^{m}=\frac{2(\lambda c)^{m+1} e^{-\lambda c}}{m !}\left[1-\frac{m+1}{4 \lambda c}+\frac{(m+1)(m-2)(2 m+3)}{144(\lambda c)^{2}}+\cdots\right]
$$


$p=1$

$$
\bar{\lambda}_{n}^{m}=\frac{2^{3}(\lambda c)^{m+2} e^{-\lambda c}}{m+1 !}\left[1-\frac{2 m+5}{2^{3} \lambda c}+\frac{m^{3}-m^{2}+34 m-54}{2^{5}(\lambda c)^{2}}+\cdots\right] .
$$

Le tableau suivant montre que ces deux relations suffisent pour les dix premières fonctions $\bar{P} e_{n}^{m}(\mu)$.

\begin{tabular}{c|c|c|c|c|c|c|c|c|c|c}
\hline \hline$n=$ & 0 & 1 & 2 & 3 & 1 & 2 & 3 & 2 & 3 & 3 \\
\hline$m=$ & 0 & 0 & 0 & 0 & 1 & 1 & 1 & 2 & 2 & 3 \\
\hline$p=$ & 0 & 0 & 1 & 1 & 0 & 1 & 1 & 0 & 0 & 0 \\
\hline
\end{tabular}

Un calcul plus poussé montre que, pour $p=0,1$ et 2 , on a

$$
\bar{\lambda}_{p}^{m}=\frac{2^{2 p+1}(\lambda c)^{m+p+1} e^{-\lambda c}}{m+p !}(1+\cdots) .
$$

Il parait probable que cette relation est générale, mais nous n'avons pas jusqu'ici pu le démontrer.

4. Nous calculerons ensuite la valeur asymptotique des constantes $\bar{\Lambda}_{n}^{m}$. Supposons tout d'abord $n-m$ pair. Avec les mêmes notations que plus haut, nous pourrons écrire (6) sous la forme

$$
y(t)=\frac{\bar{\Lambda}_{n}^{m}}{\lambda c} \int_{0}^{\infty} J_{m}\left(\left(t t_{0}\right)^{1 / 2}\right) e^{-t_{0} / 2} t_{0}^{m / 2}\left(1-t_{0} / \lambda c\right)^{-1 / 2} y\left(t_{0}\right) d t_{0} .
$$

En tenant compte de la relation bien connue suivante

$$
t L_{p}^{(m)}(t)=(2 p+m+1) L_{p}^{(m)}(t)-(p+m) L_{p-1}^{(m)}-(p+1) L_{p+1}^{(m)}(t)
$$

et de celles qui s'en déduisent, et en utilisant (3), on pourra développer la quantité $(1-t / \lambda c)^{-1 / 2} y(t)$ en série de fonctions de Laguerre. L'équation intégrale

$$
e^{-t / 2} t^{m / 2} L_{p}^{(m)}(t)=(-1)^{p} / 2 \int_{0}^{\infty} J_{m}\left(\left(t t_{0}\right)^{1 / 2}\right) e^{-t_{0} / 2 m / 2} t_{0}^{(m)} L_{p}^{(m)}\left(t_{0}\right) d t_{0}
$$

montre ensuite que le second membre est lui-même une série de fonctions de Laguerre. En identifiant les coefficients de $L_{p}^{(m)}(t)$ dans les deux membres, on trouve finalement, tous calculs faits

$$
\begin{aligned}
\bar{\Lambda}_{n}^{m}=(-1)^{p}(\lambda c / 2) & {\left[1+\frac{2 p+m+1}{2 \lambda c}\right.} \\
+ & \left.\frac{16 p^{2}+16 p m+3 m^{2}+16 p+8 m+5}{8(\lambda c)^{2}}+\cdots\right]^{-1} .
\end{aligned}
$$


Pour $n-m$ impair, on partira de la relation (7) pour obtenir

$$
y(t)=\frac{\bar{\Lambda}_{n}^{m}}{\lambda c}(1-t / \lambda c)^{-1 / 2} \int_{0}^{\infty} J_{m}\left(\left(t t_{0}\right)^{1 / 2}\right) e^{-t_{0} / 2} t_{0}^{m / 2} y\left(t_{0}\right) d t_{0}
$$

et en procédant de la même manière, on obtiendra

$$
\begin{aligned}
\bar{\Lambda}_{n}^{m}=(-1)^{p}(\lambda c / 2) & {\left[1-\frac{2 p+m+1}{2 \lambda c}\right.} \\
+ & \left.\frac{16 p^{2}+16 p m+3 m^{2}+16 p+8 m+5}{8(\lambda c)^{2}}+\cdots\right]^{-1} .
\end{aligned}
$$

5. Nous avons maintenant tout ce qu'il nous faut pour calculer $P e_{n}^{m}(0)$ et $P e_{n}^{\prime m}(0)$ au moyen de (10) et (13).

Pour vérifier numériquement l'exactitude de nos formules, nous avons comparé les valeurs qu'elles fournissent avec celles obtenues à partir des tables de J. A. Stratton et al. pour $c=8$, toujours bien entendu en adoptant la normalisation rationnelle (2).

Le tableau ci-dessous contient les résultats ainsi obtenus. L'exactitude, encore une fois, est d'autant meilleure que $n$ et $m$ sont plus petits.

\begin{tabular}{l|c|c|c|c|c|c}
\hline \multicolumn{1}{c|}{$n=$} & 0 & 1 & 2 & 1 & 2 & 2 \\
\hline \multicolumn{1}{c|}{$m=$} & 0 & 0 & 0 & 1 & 1 & 2 \\
\hline $\bar{P} e_{n}^{m}(0)$ exact & 0,00538 & 0 & $-0,0682$ & 0,0245 & 0 & 0,2479 \\
$\bar{P} e_{n}^{m}(0)$ approximatif & 0,00539 & 0 & $-0,0708$ & 0,0243 & 0 & 0,2551 \\
$\bar{P} e_{n}^{\prime m}(0)$ exact & 0 & 0,0215 & 0 & 0 & 0,1887 & 0 \\
$\bar{P} e_{n}^{\prime m}(0)$ approximatif & 0 & 0,0220 & 0 & 0 & 0,2077 & 0 \\
$\lim _{\mu=1} \bar{P}_{\left(1-\mu_{n}^{m}\right)^{m / 2} \text { approx. }} \quad$ exact & 3,863 & 2,230 & 1,559 & 8,266 & 11,098 & 38,987 \\
\hline
\end{tabular}

6. Il nous reste maintenant à obtenir la représentation asymptotique de la fonction $\bar{P} e_{n}^{m}(\mu)$ elle-même aux environs de $\mu=0$.

L'équation intégrale (5) peut s'écrire sous la forme suivante

$$
\bar{P} e_{n}^{m}(\mu)=\bar{\lambda}_{n}^{m} \int_{0}^{1}\left(e^{\lambda c \mu \mu_{0}} \pm e^{-\lambda c \mu \mu_{0}}\right)\left(1-\mu^{2}\right)^{m / 2}\left(1-\mu_{0}^{2}\right)^{m / 2} \bar{P} e_{n}^{m}\left(\mu_{0}\right) d \mu_{0}
$$

les signes + et - correspondant respectivement à $n-m$ pair et impair. On voit immédiatement que le problème se ramène au calcul de l'intégrale définie suivante 


$$
\begin{aligned}
I_{p}^{m}= & \int_{0}^{1}\left(e^{\lambda c \mu \mu_{0}} \pm e^{-\lambda c \mu \mu_{0}}\right) \\
& \cdot\left(1-\mu^{2}\right)^{m / 2}\left(1-\mu_{0}^{2}\right)^{m / 2} e^{-(\lambda c / 2)\left(1-\mu^{2}\right)} L_{p}^{(m)}\left(\lambda c\left(1-\mu_{0}^{2}\right)\right) d \mu_{0}
\end{aligned}
$$

qui peut encore s'écrire, en faisant $\mu_{0}=(1-t / \lambda c)^{1 / 2} \sim 1-t / 2 \lambda c$

$$
\begin{aligned}
I_{p}^{m}= & \frac{e^{\lambda c \mu}\left(1-\mu^{2}\right)^{m / 2}}{2(\lambda c)^{m+1}} \int_{0}^{\infty} e^{-(1+\mu) t / 2} t^{m}(1+t / 2 \lambda c) L_{p}^{(m)}(t) d t \\
& \pm \frac{e^{-\lambda c \mu}\left(1-\mu^{2}\right)^{m / 2}}{2(\lambda c)^{m+1}} \int_{0}^{\infty} e^{-(1-\mu) t / 2} t^{m}(1+t / 2 \lambda c) L_{p}^{(m)}(t) d t .
\end{aligned}
$$

En tenant compte des relations suivantes

$$
\begin{aligned}
\int_{0}^{\infty} e^{-k t} t^{m} L_{p}^{(m)}(t) d t & =\frac{p+m !}{p !} \frac{(k-1)^{p}}{k^{p+m+1}} \\
\int_{0}^{\infty} e^{-k t} t^{m+1} L_{p}^{(m)}(t) d t & =\frac{p+m !}{p !}\left[(p+m+1) \frac{(k-1)^{p}}{k^{p+m+2}}-p \frac{(k-1)^{p-1}}{k^{p+m+1}}\right]
\end{aligned}
$$

on trouve

$$
\begin{aligned}
& I_{p}^{m}=(-1)^{p}(2 / \lambda c)^{m+1} \frac{p+m !}{p !}\left(1-\mu^{2}\right)^{m / 2} \\
& \cdot\left[\phi_{p}^{m}(\mu)+\frac{p+m+1}{\lambda c} \phi_{p}^{m+1}(\mu)+\frac{p}{\lambda c} \phi_{p-1}^{m+1}(\mu)\right]
\end{aligned}
$$

en posant

$$
\phi_{p}^{m}(\mu)=2^{-1}\left[e^{\lambda c \mu} \frac{(1-\mu)^{p}}{(1+\mu)^{m+p+1}} \pm e^{-\lambda c \mu} \frac{(1+\mu)^{p}}{(1-\mu)^{m+p+1}}\right] .
$$

On déduit de ceci, en particulier, que

$$
\begin{aligned}
\phi_{p}^{m}(0) & =1 & & (n-m \text { pair }), \\
\frac{d}{d \mu}\left[\left(1-\mu^{2}\right)^{m / 2} \phi_{p}^{m}(\mu)\right]_{\mu=0} & =\lambda c & & (n-m \text { impair }) .
\end{aligned}
$$

Il est important, pour ce qui suit, de connaitre la valeur asymptotique de $I_{n}^{\prime m}(0)$. On a d'abord

$I_{p}^{\prime m}(\mu)$

$=\lambda c \int_{0}^{1}\left(e^{\lambda c \mu \mu_{0}} \pm e^{-\lambda c \mu \mu_{0}}\right)\left(1-\mu^{2}\right)^{m / 2}\left(1-\mu_{0}^{2}\right)^{m / 2} e^{-(\lambda c / 2)\left(1-\mu^{2}\right)} L_{p}^{(m)}\left(\lambda c\left(1-\mu_{0}^{2}\right)\right) \mu_{0} d \mu_{0}$ d'où 


$$
\begin{aligned}
I_{n}^{\prime m}(0) & =(\lambda c)^{-m} \int_{0}^{\infty} e^{-t / 2} t^{m} L_{p}^{(m)}(t) d t \\
& =(-1)^{p} \frac{2^{m+1}}{(\lambda c)^{m}} \frac{p+m !}{p !}
\end{aligned}
$$

résultat différent de ce que donnerait l'application directe des relations (30) et $(32,2)$.

Lorsque $\lambda c$ est suffisamment grand, on a, avec une très bonne approximation

$$
\bar{P} e_{n}^{m}(\mu)=C(\lambda c)^{m / 2} \bar{\lambda}_{n}^{m} I_{p}^{m}(\mu) .
$$

En ne conservant que les termes dominants, et en admettant que (20) est exact dans tous les cas, on aura

$$
\begin{aligned}
C & =2^{(m+1) / 2}(\lambda c)^{(m+1) / 2}\left(\frac{n+m ! p !}{(2 n+1) n-m ! p+m !}\right)^{1 / 2}, \\
\bar{\lambda}_{n}^{m} & =\frac{2^{2 p+1}(\lambda c)^{m+p+1} e^{-\lambda c}}{m+p !}
\end{aligned}
$$

et par conséquent

$$
\bar{P} e_{n}^{m}(\mu)=(-1)^{p} 2^{2 p+3 m / 2+2}(\lambda c)^{p+(m+1) / 2}
$$

$$
\cdot\left(\frac{2}{2 n+1} \cdot \frac{n+m !}{n-m !} \cdot \frac{1}{p ! p+m !}\right)^{1 / 2} e^{-\lambda c}\left(1-\mu^{2}\right)^{m / 2} \phi_{p}^{m}(\mu) .
$$

Pour avoir une idée de l'exactitude de cette formule, nous ferons le calcul pour $\mu=0, \lambda c=8$,

$$
\begin{aligned}
& m=n=p=0: \\
& \left.\quad \bar{P} e_{0}^{0}(0)=4(2 \lambda c)^{1 / 2} e^{-\lambda c}=0,005367 \text { (exact: } 0,005378\right), \\
& m=p=0, n=1: \\
& \left.\quad{ }_{P} e_{1}^{\prime 0}(0)=2(3)^{-1 / 2}(2 \lambda c)^{3 / 2} e^{-\lambda c}=0,0248 \text { (exact: } 0,0215\right), \\
& m=n=1, p=0: \\
& \quad \bar{P} e_{1}^{1}(0)=16(3)^{-1 / 2} \lambda c e^{-\lambda c}=0,02479 \text { (exact: } 0,02451 \text { ). }
\end{aligned}
$$

Etant donné qu'on n'a tenu compte que des termes dominants des développements asymptotiques et que la valeur $8 \mathrm{de} \lambda c$ n'est pas spécialement élevée, l'exactitude peut être considérée comme très bonne.

Pour le calcul effectif de la fonction $\bar{P} e_{n}^{m}(\mu)$, on utilisera la relation suivante, qui se déduit immédiatement de (30), (32.1), (32.2) et (33) 


$$
\frac{\bar{P} e_{n}^{m}(\mu)}{\bar{P} e_{n}^{m}(0)}
$$

$$
=\left(1-u^{2}\right)^{m / 2} \frac{I_{p}^{m}(\mu)+\frac{1}{2 \lambda c}\left[-\frac{(p+1)(p+2)}{4} I_{p+2}^{m}(\mu)+\cdots\right]+\cdots}{I_{p}^{m}(0)+\frac{1}{2 \lambda c}\left[-\frac{(p+1)(p+2)}{4} I_{p+2}^{m}(0)+\cdots\right]+\cdots}
$$

et on prendra pour $P e_{n}^{m}(0)$ la valeur plus exacte obtenue antérieurement. A titre d'exemple, on trouve ainsi

$$
\begin{aligned}
& \text { (35.1) } \frac{\bar{P} e_{0}^{0}(\mu)}{\bar{P} e_{0}(0)}=\frac{\phi_{0}^{0}(\mu)+\frac{1}{\lambda c} \phi_{0}^{1}(\mu)-\frac{1}{4 \lambda c} \phi_{2}^{0}(\mu)-\frac{1}{4 \lambda c} \phi_{1}^{0}(\mu)}{1+1 / 2 \lambda c} \quad(n-m=0, \text { pair }), \\
& \text { (35.2) } \frac{\bar{P} e_{1}^{0}(\mu)}{\bar{P} e_{1}^{\prime 0}(0)}=\frac{\phi_{0}^{0}(\mu)+\frac{1}{\lambda c} \phi_{0}^{1}(\mu)-\frac{1}{4 \lambda c} \phi_{2}^{0}(\mu)-\frac{1}{4 \lambda c} \phi_{1}^{0}(\mu)}{1-1 / 2 \lambda c} \quad(n-m=1, \text { impair }) .
\end{aligned}
$$

Pour $\lambda c=8, \mu=0,1$, ces relations donnent

$$
\begin{aligned}
& \frac{\bar{P} e_{0}^{0}(0,1)}{P e_{0}^{0}(0)}=1,2636 \quad(\text { exact: } 1,2584), \\
& \left.\frac{\bar{P} e_{1}^{0}(0,1)}{P{e^{\prime}}^{\prime}(0)}=0,1088 \quad \text { (exact: } 0,1087\right) .
\end{aligned}
$$

\section{BirliographiE}

1. R. Sips, Représentation asymptotique des fonctions de Mathieu et des fonctions d'onde sphéroidales, Trans. Amer. Math. Soc. vol. 66 (1949) pp. 93-134.

2. - Les constantes caractêristiques de l'équation intégrale des fonctions de Mathieu, Bull. Soc. Roy. Sci. Liège (1952) pp. 141-157.

3. E. L. Ince, Tables of the elliptic cylinder functions, Proc. Roy. Soc. Edinburgh vol. 52 (1932) pp. 355-433. 1951.

4. G. Blanch, Tables relating to Mathieu functions, New York, Columbia University Press,

5. S. Goldstein, Mathieu functions, Transactions of the Cambridge Philosophical Society vol. 23 (1927) pp. 303-336.

6. J. A. Stratton, P. M. Morse, L. J. Chu, J. D. C. Little and F. J. Corbato, Spheroidal wave functions, New York, Wiley, 1956.

Brussels, Belgium 